\title{
BMJ Open Perioperative Pain and Addiction Interdisciplinary Network (PAIN): protocol of a practice advisory for the perioperative management of buprenorphine using a modified Delphi process
}

\author{
Akash Goel, ${ }^{01,2}$ Saam Azargive, ${ }^{3}$ Joel S Weissman, ${ }^{4,5}$ Harsha Shanthanna, ${ }^{6}$ \\ Karim S Ladha, ${ }^{2,7}$ Wiplove Lamba, ${ }^{8}$ Scott Duggan, ${ }^{3}$ John G Hanlon, ${ }^{2}$ \\ Tania Di Renna, ${ }^{2,9}$ Philip Peng, ${ }^{10}$ Hance Clarke ${ }^{7}$
}

To cite: Goel A, Azargive S, Weissman JS, et al. Perioperative Pain and Addiction Interdisciplinary Network (PAIN): protocol of a practice advisory for the perioperative management of buprenorphine using a modified Delphi process. BMJ Open 2019;9:e027374. doi:10.1136/ bmjopen-2018-027374

- Prepublication history and additional material for this paper are available online. To view these files, please visit the journal online (http://dx.doi. org/10.1136/bmjopen-2018027374).

SA and JSW contributed equally.

Received 19 0ctober 2018

Revised 14 March 2019

Accepted 5 April 2019

Check for updates

(c) Author(s) (or their employer(s)) 2019. Re-use permitted under CC BY-NC. No commercial re-use. See rights and permissions. Published by BMJ.

For numbered affiliations see end of article.

Correspondence to

Dr Hance Clarke;

Hance.Clarke@uhn.ca

\section{ABSTRACT}

Introduction The ongoing opioid epidemic has necessitated increasing prescriptions of buprenorphine, which is an evidence-based treatment for opioid use disorder, and also shown to reduce harms associated with unsafe opioid administration. A systematic review of perioperative management strategies for patients taking buprenorphine concluded that there was little guidance for managing buprenorphine perioperatively. The aim of this project is to develop consensus guidelines on the optimal perioperative management strategies for this group of patients. In this paper, we present the design for a modified Delphi technique that will be used to gain consensus among patients and multidisciplinary experts in addiction, pain, community and perioperative medicine.

Methods and analysis A national panel of experts identified by perioperative, pain and/or addiction systematic review authorship established an international profile in perioperative, pain and/or addiction research, community clinical excellence and by peer referral. A steering group will develop the first round with a list of indications to be rated by the panel of national experts patients and allied healthcare professionals. In round 1 , the expert panel will rate the appropriateness of each individual item and provide additional suggestions for revisions, additions or deletions. The definition of consensus will be set a priori. Consensus will be gauged for both appropriateness and inappropriateness of treatment strategies. Where an agreement is not reached and items are suggested for addition/deletion/modification, round 2 will take place over teleconference in order to obtain consensus.

Ethics and dissemination Institutional research ethics board provided a waiver for this modified Delphi protocol. We plan on developing a national guideline for the management of patients taking buprenorphine in the perioperative period that will be generalisable across three sets of preoperative diagnoses including opioid use disorder and/or co-occurring pain disorders. The findings
Strengths and limitations of this study

Existing perioperative strategies to manage patients on buprenorphine are based on expert opinion and regional practices.

- We will employ a modified Delphi Protocol optimising medical and geographical diversity of panellists to ensure the development of a trustworthy set of guidelines.

- We will aim to include patient and allied-health care experts on our panel to ensure that the Delphi process and guideline development is patient-centred.

- Agreement and Disagreement will be measured by a priori agreed on consensus criteria.

- Given that new buprenorphine products are being released and diagnostic scales are being constantly re-evaluated, we will aim to re-visit our guidelines regularly.

will be published in peer-reviewed publications and conference presentations.

\section{INTRODUCTION}

Buprenorphine has been used for medically supervised withdrawal, historically referred to as detoxification, opioid use disorder (OUD), acute pain and chronic pain management since 2002. ${ }^{1}$ Its unique pharmacological properties and wide safety profile have made it increasingly prescribed in the patients' population with chronic pain and OUD. The number of patients on buprenorphine treatment is increasing. ${ }^{2}{ }^{3}$ Since its approval in 2002, the number of buprenorphine/naloxone tablets sold increased from 8 million in 2005 to over 145 million in 2009. 
Emerging studies have shown that increasing Medicaid coverage for buprenorphine-naloxone has resulted in an overall increase in people filing prescriptions for buprenorphine-naloxone. ${ }^{4}$

Until now, inadequate pain management is the main impetus for the perioperative discontinuation of buprenorphine. Recent evidence suggests that its perioperative discontinuation may hinder harm reduction by destabilising patients with OUD. ${ }^{5}$ For example, transitioning a patient off buprenorphine to a full agonist opioid will permit free access to opioid receptors for the purposes of analgesia, but will not address the OUD that may worsen as a result. ${ }^{5}$ Emerging evidence suggests that certain subsets of patients are less likely to experience deterioration of their OUD $^{67}$ no matter which strategy is pursued (continue or discontinue). Furthermore, there remain grave public health concerns over improper use and/or disposal of full mu-agonists that are prescribed in the perioperative period.

Currently, the quality of evidence regarding the perioperative management of patients on buprenorphine is weak. A systematic review conducted by Goel $e t a l^{8}$ revealed that the number of studies to address the perioperative dilemma is limited, and few directly evaluated the question of continuation versus discontinuation of buprenorphine..$^{9-25}$ Few studies make considerations for the possibility of relapse in cases where there has been a history of OUD. Many studies highlighted the importance of multimodal and regional anaesthesia techniques. Furthermore, the only randomised controlled trial (RCT) combined patients taking buprenorphine and methadone into one group, ${ }^{26}$ limiting the study's applicability to the important question: Should buprenorphine be continued in the perioperative period or not? It is important that the perioperative physician consider and balance the issue of pain control versus patient destabilisation. In fact, the destabilisation of a patient with an OUD risks the patient returning to the drug; this has significant negative consequences including the possibility of perioperative and postoperative overdose/death.

There is a need to develop specific guidance on how to manage OUD perioperatively. Until now, eight major guidelines ${ }^{127-33}$ were built on the backbone of anaesthesiologists' opinions and existing case reports ${ }^{9-25}$ (table 1 of the online appendix 1). Many of the existing guidelines propose discontinuation of buprenorphine before surgery, especially where high pain is expected. However, more recently, editorialised guidelines have proposed a continuation of buprenorphine depending on the preoperative dose and indication. ${ }^{33}$ Moreover, there is disagreement on the best discharge strategies for patients taking buprenorphine, irrespective of diagnosis. While most guidelines agree on major principles such as multimodal analgesia, there is no consensus on which strategies are more likely to succeed. Overall, there is disagreement on optimal preoperative, intraoperative and postoperative strategies for managing buprenorphine in patients with OUD and/or chronic pain disorders.
Given the lack of RCTs, the strength of a Delphi process is to bring geographically and medically diverse experts together and determine where there is an agreement in the perioperative management of buprenorphine. Furthermore, this process ensures the integration of multidisciplinary and patient opinions, resulting in more patient-centred and trustworthy guidelines.

\section{AIM}

We will aim to use a national expert consensus Delphibased survey technique to develop and evaluate a set of recommendations that address perioperative buprenorphine management strategies. We will seek to focus on the following factors: (1) indication for buprenorphine therapy, (2) risk of worsening of OUD and/or co-occurring pain disorder, (3) expected pain after surgery, (4) feasibility of perioperative regional anaesthesia technique, (5) utility of adjunct analgesia and (6) dose and formulation of buprenorphine therapy. We will follow the 22-step checklist recommended by the RIGHT group ${ }^{34}$ for the EQUATOR network.

\section{METHODS AND DESIGN}

This study will use a modified Delphi technique, which was developed by the RAND Corporation ${ }^{35}$ in order to address complex problems that cannot be solved without a group of experts. The Delphi technique involves quasi-anonymous voting and controlled feedback in order to generate discussion and eventual consensus on controversial topics. The Delphi method reduces the likelihood of situations in which group consensus is dominated by the perspectives of a strong minority. ${ }^{36}$

An International Research Steering Committee (Perioperative Pain and Addiction Network) has developed the list of indications, and we plan to conduct two Delphi rounds in which experts rate appropriateness of buprenorphine management. Panel responses will be de-identified, compiled, analysed and summarised before being returned to panellists. The summary report will entail qualitative and quantitative details about individual panellists' responses compared with their counterparts. It is expected that the panellist can then review their responses in light of the replies of other panellists prior to a round 2 in-person discussion and re-rating.

\section{Steering committee}

An International Steering Committee (Harvard University, University of Toronto, McMaster University and Queen's University) was formed to develop and conduct this project and consists of representation from various disciplines (Anaesthesiology, Family Practice, Epidemiology, Addictions Medicine and Pain Medicine), geographical areas (Canada and USA) and research expertise (Delphi, health services and quantitative methods), referred to collectively as the Perioperative Pain and Addiction Interdisciplinary Network (PAIN). 
A literature review including a systematic review was conducted by the steering committee to understand the scope of management strategies published to date. The protocol and associated methods were established and agreed on through in-person, telephone and email communication. Important functional domains of the research question were considered by the steering committee after completion of the literature review (ie, preoperative management, postoperative buprenorphine management, inpatient use of opioids and adjuncts for analgesia, involvement of outpatient providers and discharge planning). These domains are represented as 'sections' in the final questionnaire. Reference was made to previous and published Delphi studies. ${ }^{36}{ }^{37}$ There were three drafts reviewed by the Steering committee, and a final draft after a self-test by the steering committee provided a further set of comments and suggestions.

\section{Generation of the chapters with indications (items)}

The indications and domains identified from the systematic review ${ }^{8}$ were examined along with the available evidence from existing recommendations on this topic. ${ }^{127-33}$ Furthermore, case experience from addiction and pain physicians was used to complete a panel rater form based on the RAND questionnaires used in existing studies. ${ }^{35}$ The form was generated in order to reflect the essential processes involved in the perioperative experience of patients maintained on buprenorphine. In total, 840 indications will be divided into three chapters of preoperative diagnoses. (1) OUD only, (2) OUD with co-occurring pain disorder, (3) pain disorder only. Panellists will indicate their preference for various perioperative strategies (continue, reduce and stop) by systematically rating these indications from chapters 1 to 3 .

\section{Selection of national panel of experts (participants and recruitment)}

'Experts' were defined as individuals involved in the management, development, research, teaching or analysis of clinical perioperative buprenorphine strategies. Because the Delphi group size depends more on optimising group dynamics to obtain consensus than statistical analysis, we aimed for a panel size based on original Delphi methodology from the RAND study ${ }^{35}$ (optimal panel size of 9 and no $>15$ ). We set an a priori estimate of panellist attrition rate (20\%) and aimed to select up to 15 panellists for round 1 (the maximum recommended by the RAND authors).

To identify experts in the field of addiction and perioperative medicine, we reviewed authorship of published guidelines and case reports of buprenorphine management in the perioperative period; we identified established national and regional profiles in addiction, pain or perioperative medicine; we solicited peer recommendations from individuals on boards of the National Canadian Pain, Addiction, and Anaesthesiology Societies (CSAM, CPS and CAS). We sought to diversify our panel by selecting panellists with practice experience in all the Canadian provinces, membership on professional societies and wide-ranging expertise.

We initially reached out to these prospective expert panellists by emailing solicitation letters describing the project and the timelines involved (online appendix $2)$. Prospective panellists were then asked to complete and return a conflict of interest form (online appendix 3) along with their indication of interest in the project. Conflicts of interest were reviewed by the Steering Committee and prospective panellists with potential conflicting industry affiliations were removed from the final shortlist. Possible incentives for participation in this process included $^{1}$ the opportunity to be selected into a diverse group ${ }^{2}$ unique educational opportunities and $^{3}$ increased internal and external visibility. ${ }^{1}$

\section{Patient and public involvement}

We included a patient on our steering committee and an expert panel in order to develop a research question and outcome measures that were informed by patient priorities, experience and preferences. Furthermore, the inclusion of a nurse practitioner also allowed the panellists to consider the values and preferences of the target population.

\section{Ethics}

All participants will be informed that by responding to the questionnaire, they have indicated their consent to participate in the study and have their de-identified responses included in associated analyses. All data will be preserved on paper (under lock and key) as well as a computer (which is password protected) in a locked office, in accordance with standard guidelines. Only the steering committee will have access to the data, which will be destroyed after 5 years in accordance with local guidelines.

We obtained an official waiver from our institutional research ethics board (REB) for the conduct of this protocol. The REB deemed our Delphi protocol not to be research as defined in the Tri-Council Policy Statement, and therefore, did not fall under the purview of the REB.

\section{Delphi procedures (data collection and data analysis)}

We will administer paper questionnaires that will be delivered as attachments to expert panellists by email. Panellists will be asked to print out the questionnaires and complete them on paper. The email will include an instruction form (online appendix 4) that includes a table of contents and a sample exercise grid. Furthermore, a systematic review completed by the steering committee will be provided to panellists as a resource to supplement existing knowledge and experience in this topic. To reduce the likelihood of attrition bias, we will notify the panel that authorship of the final guideline document will be offered only to participants who complete the entire Delphi process. 


\section{Rounds 1 and 2 of Delphi procedure}

The Delphi process will consist of two survey rounds. The first round will consist of questionnaires that will be completed remotely by all panellists. Panellists will be blinded to each other's participation in the first round to prevent any communication that may lead to bias in the ratings. The first survey round will extend to 3 weeks, with the first week dedicated to addressing any concerns raised by panellists. In this round, panellists will first be asked to rate the appropriateness of continuing or stopping buprenorphine where: 1=veryappropriate to stop buprenorphine and 9=veryappropriate to continue buprenorphine at the same or reduced dose. Next, panellists will be asked to rate the appropriateness of reducing versus maintaining dosage, where: $1=$ =very appropriate to reduce buprenorphine dosage; and $9=$ very appropriate to continue buprenorphine at the same dosage. Panellists will also be asked to identify potential deletions, modifications, or points of clarification on return of the rating forms. Furthermore, they will be urged to consider patient populations that will require individualised approaches to the management of their buprenorphine dose in the perioperative period. Panellists will then scan and return their rating forms with unique personal identifier codes on each page.

In order to identify thresholds for agreement, we will include predetermined information about buprenorphine dose, formulation, diagnosis (pain and/or OUD), the risk of exacerbation of underlying disorder, expected surgical pain and availability of regional anaesthetic technique in the final list of indications. These 840 indications will reflect the complete perioperative period, including strategies for communication with the outpatient provider and utilisation of multimodal analgesia.

After completion of round 1, a 2-week analysis period will ensue, in which two blinded independent analysts will extract de-identified data from rating forms and input data into two mutually exclusive databases. De-identified results including scores for each indication (including median and mode scores, interquartile ranges, indications with universal consensus and qualitative feedback) along with a narrative report of the findings will be remitted to individual panellists to review prior to round 2 of the Delphi process. The second-round meeting will be conducted in person and over teleconference given the geographic diversity of expert panellists. Any ambiguous indications or external factors not previously considered will be aggregated for discussion during this round. Panellists will have the opportunity to discuss the addition or removal of indications (items) at this point. If indications are deemed to be insufficient (not capturing the breadth of the theme) or overly inclusive (extreme granularity of indications) then the steering committee will offer a second round of rating after inclusion or exclusion of culprit items. If duplicate indications exist, where possible, the steering committee will aim to combine indications.

\section{Definition of consensus}

In any Delphi process, decision rules are determined in advance to both define and determine consensus. Consensus on a topic is usually determined if a certain number or percentage of the votes fall within a prescribed range. The Steering Committee has a priori decided on its definition of consensus in order to avoid bias. Using the European Union BIOMED Concerted Action on Appropriateness for surgical procedures as referenced in the RAND/UCLA Appropriateness Method User's Manual. ${ }^{35}$

We define consensus (agreement) in two ways: (1) appropriate treatment defined as a clustering of scores with a median score in the high end of the scale without 'disagreement' (ie, more than two panellists' scores in the low end of the scale, 1-3) and (2) inappropriate treatment defined as a clustering of scores with a median score in the low end of the scale without 'disagreement' (ie, more than two panellists' scores in the high end of the scale, 7-9).

\section{Development of guidelines and recommendations}

A final operational manual with decision rules for each indication will be presented to panellists during the second-round meeting, with accompanying explanatory documents as necessary. Panellists will be asked to rank and order the recommendations to rationalise the number of items included in the final guideline as per the EQUATOR network's reporting tool for practice guidelines in healthcare (RIGHT).$^{34}$ An email questionnaire will aim to obtain a final majority agreement on the synthesis of comments after the second round of the Delphi process. It is expected that a clear and concise rationale will accompany individual recommendation statements.

\section{Review and quality assurance}

We plan to use a two-step process in order to develop and refine an internationally agreed on guideline for the perioperative management of patients maintained on buprenorphine. Initially, a draft guideline will undergo an independent review by members external to the steering committee. Any comments will be addressed explicitly in the final guideline document. A questionnaire will be emailed out to panellists after the second round to solicit suggestions for improvement in future iterations.

The guideline document should reflect the needs of patients who have co-occurring disorders where possible, therefore facilitating its use in as many perioperative scenarios as possible. The final consensus guidelines will be submitted to a perioperative journal and championed by individual panellists at their home institutions.

To test the acceptability of the proposed guidelines due to varying geography and practice patterns, we will seek annual comments and suggestions from regional and national users of the guideline. The guideline document should be reviewed annually in order to reflect shifting evidence and expert opinion. 


\section{Funding and management of interests}

No funding sources will be used in any stage of the guideline development. Individual steering committee members and panellists were made to complete conflict of interest forms prior to involvement in this process. Any prospective steering committee members or panellists with perceived conflicts of interests were not included at any point of the guideline development. Original declaration forms outlining conflict of interest are available upon request to the first author of the study (AG).

\section{Limitations of the guideline}

Increasingly, providers are beginning to see off-label prescription of sublingual buprenorphine for patients with pain disorders. Furthermore, there are several new formulations of buprenorphine emerging. As evidence emerges and new formulations of buprenorphine are developed, these guidelines will require updating in the future hopefully on an annual basis.

\section{Delphi study status}

The first round of the Delphi process will begin in October, with an in-person, second round meeting scheduled in November 2018. Data collection and analysis will occur after the second-round meeting if panellists and the steering committee are satisfied that all important questions have been addressed. A paper reporting the results of the Delphi process will be submitted for publication in early 2019 followed by conference presentations. Data collection will start in October 2018 and anticipated to be completed by December 2018.

\section{Author affiliations \\ ${ }^{1}$ Department of Epidemiology, Harvard University T H Chan School of Public Health, Boston, Massachusetts, USA \\ ${ }^{2}$ Department of Anesthesia, University of Toronto, Toronto, Ontario, Canada ${ }^{3}$ Department of Anesthesiology and Perioperative Medicine, Queens University School of Medicine, Kingston, Ontario, Canada \\ ${ }^{4} \mathrm{TH}$ Chan School of Public Health, Harvard University, Boston, Massachusetts, USA ${ }^{5}$ Center for Surgery and Public Health, Brigham and Women's Hospital, Boston, Massachusetts, USA \\ ${ }^{6}$ Department of Anesthesia, McMaster University St Joseph's Health Care, Hamilton, Ontario, Canada \\ ${ }^{7}$ Pain Research Unit, Toronto General Hospital, University Health Network, Toronto, Ontario, Canada \\ ${ }^{8}$ Department of Psychiatry, University of Toronto, Toronto, Ontario, Canada \\ ${ }^{9}$ Toronto Academic Pain Medicine Institute, Women's College Hospital, Toronto, Ontario, Canada \\ ${ }^{10}$ Toronto Western Hospital, University of Toronto, Toronto, Ontario, Canada}

Acknowledgements The authors would like to acknowledge Michael SatokWolman (our patient advisor) in his contributions to the development of rater forms and the Delphi process. They would like to acknowledge Kari Van-Kamp (our allied health care representative) in her contributions as to the development of the Delphi protocol.

Contributors AG, JSW and HS: developed the Delphi Protocol and methodology. $A G$ and $S A$ : developed and modified Round 1 panel rating forms. AG, KL, WL, SD, $\mathrm{JH}, \mathrm{TDR}, \mathrm{PWP}$ and HC: involved in the development of the research question and formulation of the Delphi Protocol as part of the Steering Committee.

Funding HS is supported by the Canadian Anesthesia Research Foundation grant as a 'Career Investigator Award'. HC and KL are supported by the Ontario Ministry of Health and Long Term Care and by Merit Awards by the Department of Anaesthesia,
University of Toronto. Funding was provided by the Ontario Ministry of Health and Long Term Care

Competing interests None declared.

Provenance and peer review Not commissioned; externally peer reviewed.

Open access This is an open access article distributed in accordance with the Creative Commons Attribution Non Commercial (CC BY-NC 4.0) license, which permits others to distribute, remix, adapt, build upon this work non-commercially, and license their derivative works on different terms, provided the original work is properly cited, appropriate credit is given, any changes made indicated, and the use is non-commercial. See: http://creativecommons.org/licenses/by-nc/4.0/.

\section{REFERENCES}

1. Anderson TA, Quaye ANA, Ward EN, et al. To Stop or Not, That Is the Question: Acute Pain Management for the Patient on Chronic Buprenorphine. Anesthesiology 2017;126:1180-6.

2. Potter JS, Dreifuss JA, Marino EN, et al. The multisite prescription opioid addiction treatment study: 18-month outcomes. Journal of Substance Abuse Treatment 2015;1:62-9.

3. Weiss RD, Potter JS, Griffin ML, et al. Long-term outcomes from the National Drug Abuse treatment clinical trials network prescription opioid addiction treatment study. Drug Alcohol Depend 2015;150:112-9.

4. Saloner B, Levin J, Chang HY, et al. Changes in BuprenorphineNaloxone and Opioid Pain Reliever Prescriptions After the Affordable Care Act Medicaid Expansion. JAMA Netw Open 2018;1:e181588.

5. Ward EN, Quaye AN, Wilens TE. Opioid use disorders: perioperative management of a special population. Anesth Analg 2018;127:539-47.

6. Adams E, Sharifi N. Lappalainen L: a guideline for the clinical management of opioid use disorder: British Columbia Centre on Substance Use. 2017:18-19 http://www.bccsu.ca/care-guidancepublications (Last accessed 4 Dec 2017).

7. Cornish R, Macleod J, Strang J, et al. Risk of death during and after opiate substitution treatment in primary care: prospective observational study in UK General Practice Research Database. BMJ 2010;341:c5475.

8. Goel A, Azargive S, Lamba W, et al. The perioperative patient on buprenorphine: a systematic review of perioperative management strategies and patient outcomes. Can J Anaesth 2019;66:201-17.

9. Huang A, Katznelson R, de Perrot M, et al. Perioperative management of a patient undergoing Clagett window closure stabilized on Suboxone ${ }^{\circledR}$ for chronic pain: a case report. Can $J$ Anaesth 2014;61:826-31.

10. Book SW, Myrick H, Malcolm R, et al. Buprenorphine for postoperative pain following general surgery in a buprenorphinemaintained patient. Am J Psychiatry 2007;164:979.

11. Silva MJ, Rubinstein A. Continuous perioperative sublingual Buprenorphine. J Pain Palliat Care Pharmacother 2016;30:289-93.

12. Chern SY, Isserman R, Chen L, et al. Perioperative Pain Management for Patients on Chronic Buprenorphine: A Case Report. J Anesth Clin Res 2013;3.

13. Israel JS, Poore SO. The clinical conundrum of perioperative pain management in patients with opioid dependence: lessons from two cases. Plast Reconstr Surg 2013;131:657.

14. Jones HE, Johnson RE, Milio L. Post-cesarean pain management of patients maintained on methadone or buprenorphine. Am J Addict 2006;15:258-9.

15. Marcucci C, Fudin J, Thomas $P$, et al. A new pattern of buprenorphine misuse may complicate perioperative pain control. Anesth Analg 2009;108:1996-7.

16. Khelemsky Y, Schauer J, Loo N. Effect of buprenorphine on total intravenous anesthetic requirements during spine surgery. Pain Physician 2015;18:261-4.

17. McCormick Z, Chu S, Chang-Chien G. Joseph P: Acute pain control challenges with buprenorphine/naloxone therapy in a patient with compartment syndrome secondary to McArdle's Disease: a case report and review. Pain Physician 2013;14:1187-91.

18. Rodgman C, Pletsch G. Double successful buprenorphine/naloxone induction to facilitate cardiac transplantation in an iatrogenically opiate-dependent patient. J Addict Med 2012;6:177-8.

19. Kornfeld $H$, Manfredi L. Effectiveness of full agonist opioids in patients stabilized on buprenorphine undergoing major surgery: a case series. Am J Ther 2010;17:523-8.

20. Hassamal S, Goldenberg M, Ishak W, et al. Overcoming barriers to initiating medication-assisted treatment for heroin use disorder in a 
general medical hospital: a case report and narrative literature review. J Psychiatr Pract 2017;23:221-9.

21. Brummett CM, Trivedi KA, Dubovoy AV, et al. Dexmedetomidine as a novel therapeutic for postoperative pain in a patient treated with buprenorphine. J Opioid Manag 2009;5:175-9.

22. Gupta $D$, Christensen $C$, Soskin V. Marked variability in peri-partum anesthetic management of patients on buprenorphine maintenance therapy (BMT): can there be an underlying acute opioid induced hyperalgesia precipitated by neuraxial opioids in BMT patients? Middle East J Anaesthesiol 2013;22:273-81.

23. Macintyre PE, Russell RA, Usher KA, et al. Pain relief and opioid requirements in the first 24 hours after surgery in patients taking buprenorphine and methadone opioid substitution therapy. Anaesth Intensive Care 2013;41:222-30.

24. Meyer M, Paranya G, Keefer Norris A, et al. Intrapartum and postpartum analgesia for women maintained on buprenorphine during pregnancy. Eur J Pain 2010;14:939-43.

25. Hansen LE, Stone GL, Matson CA, et al. Total Joint Arthroplasty in patients taking Methadone or Buprenorphine/Naloxone preoperatively for prior heroin addiction: a prospective matched cohort study. J Arthroplasty 2016;31:1698-701.

26. Höflich AS, Langer M, Jagsch R, et al. Peripartum pain management in opioid dependent women. Eur J Pain 2012;16:574-84.

27. Childers JW, Arnold RM. Treatment of pain in patients taking buprenorphine for opioid addiction \#221. J Palliat Med 2012;15:613-4.

28. Bryson EO. The perioperative management of patients maintained on medications used to manage opioid addiction. Curr Opin Anaesthesiol 2014;27:359-64.
29. Berry P, Besio S, Brooklyn J, et al. Vermont Practice Guidelines. : Vermont Department of health; division of alcohol and drug abuse programs; office of Vermont health access, 2015:30: 1.

30. Sen S, Arulkumar S, Cornett EM, et al. New pain management options for the surgical patient on Methadone and Buprenorphine. Curr Pain Headache Rep 2016;20:16.

31. Brummet C. Management of sublingual buprenorphine in the acute postoperative setting: The University of Michigan Health System, 2008.

32. Jonan $A B$, Kaye AD, Urman RD. Buprenorphine formulations: clinical best practice strategies recommendations for perioperative management of patients undergoing surgical or interventional pain procedures. Pain Physician 2018;21:E1-E12.

33. Lembke A, Ottestad E, Schmiesing C. Patients maintained on Buprenorphine for opioid use disorder should continue Buprenorphine Through the perioperative period. Pain Med 2019;20:425-8.

34. Chen $\mathrm{Y}$, Yang $\mathrm{K}$, Marušic A, et al. A reporting tool for practice guidelines in health care: the RIGHT statement. Ann Intern Med 2017;166:128-32.

35. Fitch K, Bernstein SJ, Aguilar MD, et al. The RAND/UCLA Appropriateness method user's manual Santa Monica. CA: Rand Health, 2001.

36. Slade SC, Dionne CE, Underwood M, et al. Standardised method for reporting exercise programmes: protocol for a modified Delphi study. BMJ Open 2014;4:e006682.

37. Okoli C, Pawlowski SD. The Delphi method as a research tool: an example, design considerations and applications. Inf Manage 2004;42:15-29. 